\title{
Scalable Production of Graphene-Based Wearable E-Textiles
}

Nazmul Karim, ${ }^{* \dagger, \perp}$ @ Shaila Afroj, ${ }^{\dagger, \ddagger, \perp}$ Sirui Tan, ${ }^{\S}$ Pei He, ${ }^{\S}$ Anura Fernando, ${ }^{\S}$ Chris Carr, and Kostya S. Novoselov ${ }^{\dagger,+}$

${ }^{\dagger}$ The National Graphene Institute (NGI), The University of Manchester, Booth Street East, Manchester M13 9PL, United Kingdom ${ }^{\ddagger}$ School of Physics and Astronomy, The University of Manchester, Oxford Road, Manchester M13 9PL, United Kingdom

${ }^{\S}$ School of Materials, The University of Manchester, Oxford Road, Manchester M13 9PL, United Kingdom

"School of Design, The University of Leeds, Leeds LS2 9JT, United Kingdom

\section{Supporting Information}

ABSTRACT: Graphene-based wearable e-textiles are considered to be promising due to their advantages over traditional metal-based technology. However, the manufacturing process is complex and currently not suitable for industrial scale application. Here we report a simple, scalable, and cost-effective method of producing graphenebased wearable e-textiles through the chemical reduction of graphene oxide (GO) to make stable reduced graphene oxide (rGO) dispersion which can then be applied to the textile fabric using a simple pad-dry technique. This application method allows the potential manufacture of

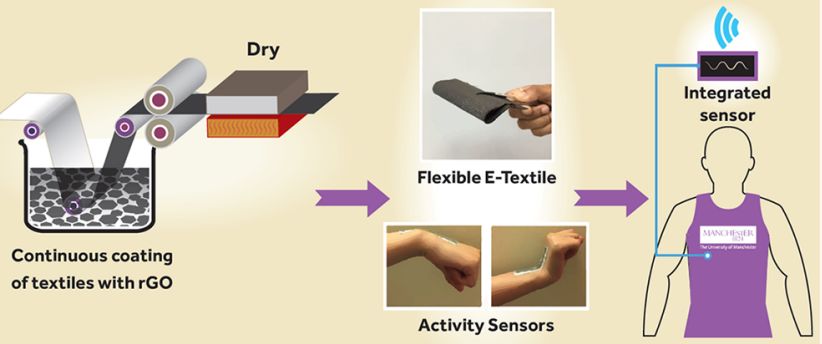
conductive graphene e-textiles at commercial production rates of $\sim 150 \mathrm{~m} / \mathrm{min}$. The graphene e-textile materials produced are durable and washable with acceptable softness/hand feel. The rGO coating enhanced the tensile strength of cotton fabric and also the flexibility due to the increase in strain\% at maximum load. We demonstrate the potential application of these graphene e-textiles for wearable electronics with activity monitoring sensor. This could potentially lead to a multifunctional single graphene e-textile garment that can act both as sensors and flexible heating elements powered by the energy stored in graphene textile supercapacitors.

KEYWORDS: graphene, wearables, e-textiles, textile sensors, activity monitoring

M ultifunctional wearable e-textiles are becoming increasingly popular since such technology makes life safer, healthier, and more comfortable. ${ }^{1}$ This technology allows the production of highly innovative and intelligent e-textile garments that can perform as a sensor, actuator, power generator, and energy storage device all at the same time. ${ }^{2}$ The market for such e-textiles has been growing rapidly and is forecasted to grow up to USD 5 billion by 2027 through the integration of lightweight and flexible electronics into everyday garment. ${ }^{3}$ However, the challenge to achieving this goal lies within the complex and time-consuming manufacturing process of e-textiles and the use of expensive, ${ }^{4}$ toxic, ${ }^{5}$ and nonbiodegradable, ${ }^{6}$ not very stable metallic conductive materials such as silver $(\mathrm{Ag})$ and copper $(\mathrm{Cu})$.

Graphene has opened up a wide range of flexible electronics applications due to its outstanding electrical, mechanical, and other performance properties. ${ }^{7-9}$ The unique properties of graphene are mostly associated with the individual sheets. Therefore, it is important for industrial applications that bulk amounts of graphene can be produced in a processable form that does not agglomerate. However, graphene sheets, unless well separated from each other, have a tendency to agglomerate and even restack to form graphite through van der Waals interactions. ${ }^{10}$ The graphite oxide route is currently the most popular wet chemical method for producing scalable quantities of graphene materials such as graphene oxide $(\mathrm{GO})^{11}$ and reduced graphene oxide ( $\mathrm{rGO}) .{ }^{12}$ This is because of its higher yield, good colloidal stability, and excellent dispersibility in various solvents. ${ }^{13-15}$

Graphene-based materials, such as GO and rGO, have already shown great promise by being fabricated into environmental friendly wearable e-textiles. ${ }^{16-21}$ The presence of the negative charge in GO not only helps to form stable dispersions ${ }^{14}$ but also allows their interaction with the functional groups of the fibers/fabrics. ${ }^{20,22}$ Thus, it provides better fixation with textile fabrics and produces flexible, washable and durable wearable e-textiles. Several techniques

Received: August 19, 2017

Accepted: November 29, 2017

Published: November 29, 2017 


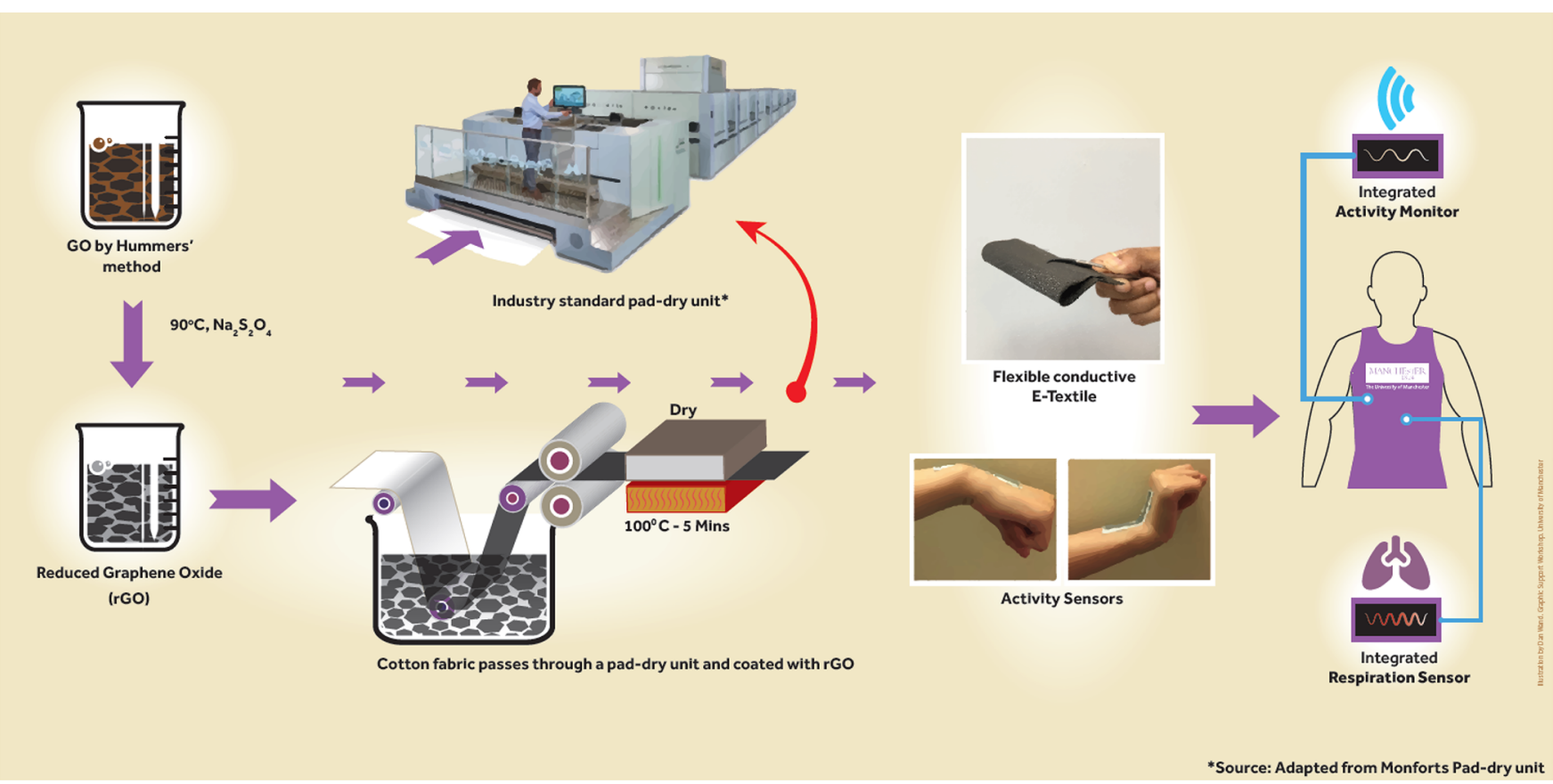

Figure 1. Schematic diagram of the scalable production of graphene-based wearable e-textiles. Illustration by Daniel Wand and used with permission from the artist.

have been used to coat textiles with graphene materials such as dip coating, ${ }^{23}$ vacuum filtration, ${ }^{24}$ brush coating, ${ }^{25}$ direct electrochemical deposition, ${ }^{26}$ electrophoresis, ${ }^{27}$ kinetic trapping method, ${ }^{28}$ wet transfer of monolayer, ${ }^{29}$ or screen printing. ${ }^{30}$ However, these are time-consuming and multi-stage manufacturing processes; not suitable for large-scale production. Therefore, there is a need for a low-cost, continuous, and scalable process for fabricating commercial wearable e-textiles.

The traditional method of coating textiles with rGO usually goes via coating textiles with GO first, followed by subsequent reduction by partial restoration of the $\mathrm{sp}^{2}$ structure. ${ }^{31}$ This can be achieved by chemical, ${ }^{23}$ thermal, $^{32}$ or electrochemical reduction ${ }^{17}$ methods. Thermal reduction of GO coated textiles has also been reported; ${ }^{19}$ however the textile fabrics are temperature sensitive and lose strength significantly at higher temperature. ${ }^{33}$ The electrochemical reduction requires conductive substrates to carry current flow, which is suitable for carbon cloths only. The majority of graphene e-textiles studies concentrate on chemical reductants such as ascorbic acid, ${ }^{18}$ hydrazine, ${ }^{27}$ hydroiodic acid, ${ }^{34}$ sodium borohydride, ${ }^{23}$ and sodium hydrosulphite ${ }^{22}$ in the post-treatment process, where GO coated fabrics are reduced to electrically conductive rGO etextiles. However, most of these reducing agents are toxic and may not be suitable for e-textiles application which would be in contact with human body. The other potential reducing agents are either not very efficient or require longer processing time to reduce the GO. ${ }^{35}$ Recently, Shateri-Khalilabad et al. ${ }^{23}$ reported using of $\mathrm{Na}_{2} \mathrm{~S}_{2} \mathrm{O}_{4}$ as an efficient reducing agent to reduce GO coated textiles. However, their process involves multiple "dip and dry" coating with $\mathrm{GO}$ and post-reduction with $\mathrm{Na}_{2} \mathrm{~S}_{2} \mathrm{O}_{4}$, which is time-consuming and requires a number of cleaning steps. Therefore, it is desirable to have an efficient and scalable reduction process for manufacturing rGO-based wearable etextiles.

Here we report a simple, scalable, and cost-effective method of producing graphene-based e-textiles. In contrast with previous approaches, we reduce GO in solution, chemically functionalize it, and use the resulting functionalized rGO to coat the textiles. As illustrated in Figure 1, we use a modified Hummers method first to synthesize GO and then chemically reduce this in situ using $\mathrm{Na}_{2} \mathrm{~S}_{2} \mathrm{O}_{4}$, an efficient and commercially available reducing agent for textile materials. During in situ chemical reduction of GO, we also incorporate poly(styrenesulfonate) (PSS) in order to functionalize the surface of $\mathrm{rGO}$ to form a stable dispersion. We use this $\mathrm{rGO}$ dispersion to coat textile fabrics using a simple and continuous pad-dry process and also demonstrate the application of these graphene e-textiles via associated activity monitoring sensor.

\section{RESULTS AND DISCUSSION}

Scalable Production of Stable rGO Dispersion and Characterization. We synthesized GO using a simple and cheap solution process, that is, a modified Hummers method. $^{36,37}$ In this process, the oxidative intercalation and production of oxygen-containing functional groups on the graphene layers helps to disperse and stabilize GO sheet in an aqueous medium. ${ }^{38}$ As prepared, GO was chemically reduced to $\mathrm{rGO}$ using sodium hydrosulphite $\left(\mathrm{Na}_{2} \mathrm{~S}_{2} \mathrm{O}_{4}\right)$ as the reducing agent. $\mathrm{Na}_{2} \mathrm{~S}_{2} \mathrm{O}_{4}$ is widely used in the textile industry for efficient, reliable, and rapid reduction of vat dyes in order to solubilize the pigment molecule. ${ }^{39}$ The structure of GO is similar to that of vat dyes used in the textile industry (Figure S1, Supporting Information), as oxygen functional groups are covalently attached to their aromatic benzene rings. ${ }^{40}$ With the addition of $\mathrm{Na}_{2} \mathrm{~S}_{2} \mathrm{O}_{4}$ to the yellow-brown dispersion of GO, the color of the dispersion turns opaque black almost immediately (Figure S2, Supporting Information), which confirms the efficient reduction of GO. This is in agreement with previous studies, ${ }^{40,41}$ where a change of color was observed within 15$20 \mathrm{~min}$. As dye reduction with $\mathrm{Na}_{2} \mathrm{~S}_{2} \mathrm{O}_{4}$ has already been commercially used for many years in the textile industry, we therefore believe it has significant commercial potential to be used for stable rGO dispersion production. 

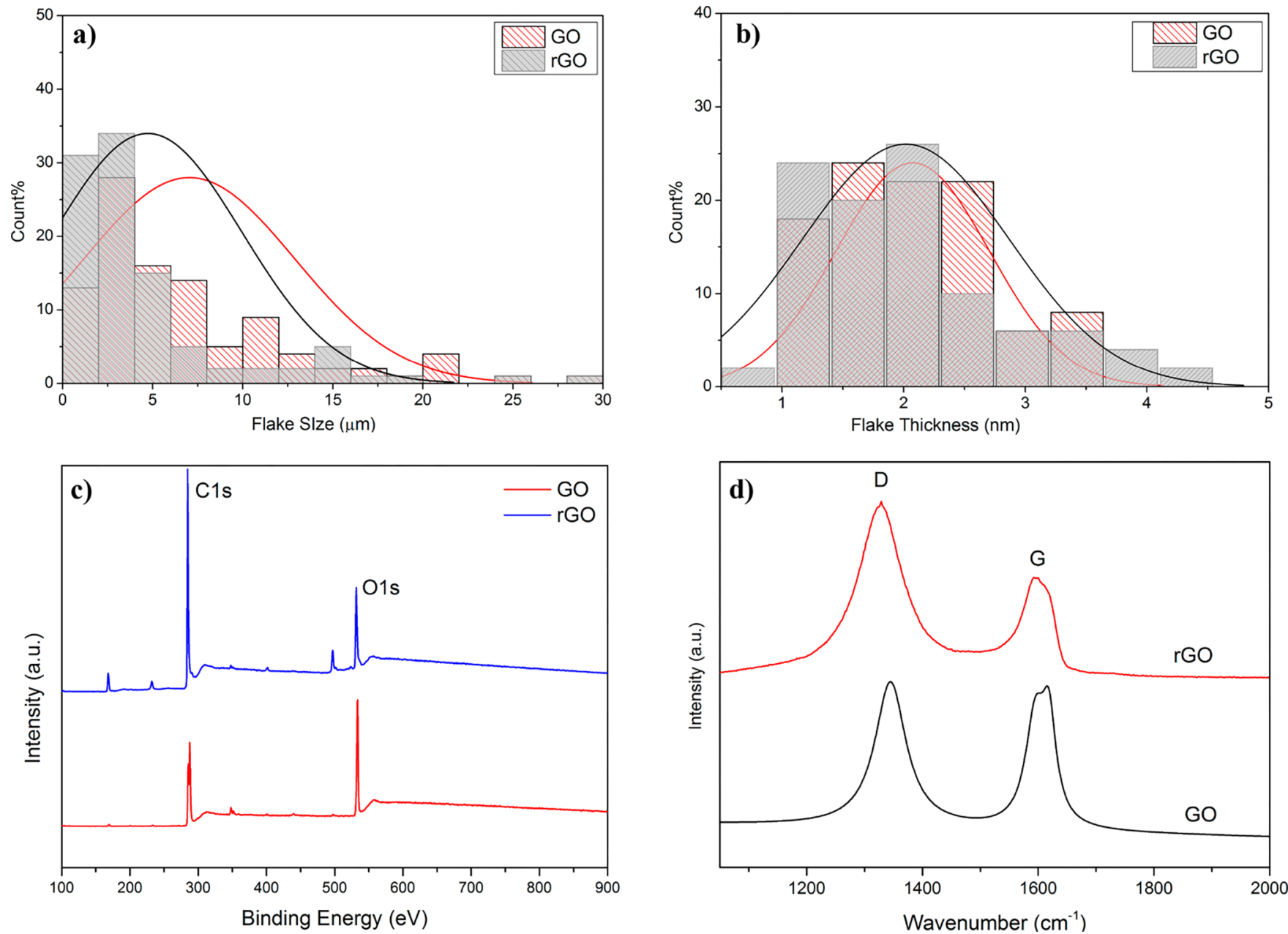

Figure 2. (a) Flake size distribution of GO and rGO. (b) Flake thickness distribution of GO and rGO. (c) Wide scan XPS spectra of GO and rGO. (d) Raman spectra of GO and rGO.

Shateri-Khalilabad et al. ${ }^{23}$ used a number of reducing agents such as $\mathrm{NaBH}_{4}, \mathrm{~N}_{2} \mathrm{H}_{4}, \mathrm{C}_{6} \mathrm{H}_{8} \mathrm{O}_{6}, \mathrm{Na}_{2} \mathrm{~S}_{2} \mathrm{O}_{4}$, and $\mathrm{NaOH}$ to reduce $\mathrm{GO}$ coated fabrics. However, they found that $\mathrm{Na}_{2} \mathrm{~S}_{2} \mathrm{O}_{4}$ was the best reducing agent to achieve higher electrical conductivity in the e-textiles. Zhou et al. ${ }^{40}$ explained the reduction mechanism of $\mathrm{Na}_{2} \mathrm{~S}_{2} \mathrm{O}_{4}$ which has a low electrode potential $\left(E^{\circ} \mathrm{SO}_{3}{ }^{2-}\right.$ / $\left.\mathrm{S}_{2} \mathrm{O}_{4}{ }^{2-}=-1.12 \mathrm{~V}\right)$ in alkaline conditions and dissociates two protons to become a nucleophile. This nucleophile attacks the epoxide and hydroxyl groups of GO with a back side $S_{N} 2$ nucleophilic reaction to form an intermediate. Reduced graphene oxide is formed due to hydro-thermal reduction, and the reducing agent $\left(\mathrm{S}_{2} \mathrm{O}_{4}{ }^{2-}\right)$ is oxidized to sulphite $\left(\mathrm{SO}_{3}{ }^{2-}\right)$.

Although the reduction of graphene oxide is a popular approach, the major problem associated with this route is that rGO aggregates in aqueous solution due to its hydrophobic nature, especially at higher concentration. To prevent restacking of graphene flakes, surface characteristics should be modified by the addition of surface functionality through either covalent bonding or noncovalent interactions. ${ }^{42,43}$ Covalent bonding is commonly preferred when stability and strong mechanical properties of modified graphene are required. In this study, we functionalized the rGO surface using poly(styrenesulfonate) (PSS), which improved the dispersibility and stability of the rGO in aqueous media. ${ }^{44}$ The dispersion (3.2 $\mathrm{mg} / \mathrm{mL}$ ) was stable for more than 6 months, and moreover, the
PSS functionalized rGO dispersion offers better wettability ${ }^{45}$ and enables better adhesion to the fibrous substrates.

Field emission gun scanning electron microscopy (FEGSEM) was used to assess the lateral size of precursor GO and resultant rGO composite ink. Figure 2a shows a statistical analysis of 100 flakes where the mean lateral dimension of GO was $5.85 \mu \mathrm{m}$ and that of $\mathrm{rGO}$ was $4.86 \mu \mathrm{m}$. The decreased flake size of GO after reduction may be due to the stresses it was subjected to during pre-mixing and centrifugation steps in postwashing cycles. Figure $2 \mathrm{~b}$ shows flake thickness $(h)$ of GO and $\mathrm{rGO}$, which was $2.07 \mathrm{~nm}$ for GO and $2.21 \mathrm{~nm}$ for rGO. Figure $2 \mathrm{~b}$ indicates that the distribution was shifted toward higher $h$ for $\mathrm{rGO}$, which may be due the presence of cross-linking polymer (PSS) covering the graphene flake. ${ }^{44}$

XPS analysis provides further evidence of efficient reduction by $\mathrm{Na}_{2} \mathrm{~S}_{2} \mathrm{O}_{4}$, Figure $2 \mathrm{c}$, as the wide scan XPS spectrum shows a significant increase in carbon content after reduction. The $\mathrm{C} / \mathrm{O}$ ratio was also increased from $2.4(\mathrm{GO})$ to $6.6(\mathrm{rGO})$ after reduction (Table 1$)$. Moreover the $\mathrm{C}(1 \mathrm{~s})$ spectra of $\mathrm{GO}$ and rGO (Figure S3) demonstrates that the peaks associated with oxygen functional groups ${ }^{46}$ sharply diminished after GO reduction to $\mathrm{rGO}$, with a small amount of residual oxygen functionality evident at $286.5 \mathrm{eV}$. The $\mathrm{C}(1 \mathrm{~s})$ spectrum of rGO was almost identical to graphite or graphene, which exhibited clear restoration of graphitic structure through chemical reduction. ${ }^{47}$ Figure $2 \mathrm{~d}$ shows the Raman spectra of GO and 
Table 1. Wide Scan XPS Analysis C/O Ratio

\begin{tabular}{lccc}
\multicolumn{1}{c}{ sample } & C (\%) & O (\%) & C/O \\
GO & 70.7 & 29.4 & 2.4 \\
rGO & 86.8 & 13.2 & 6.6 \\
untreated cotton & 73.9 & 26.1 & 2.8 \\
rGO coated (padded) cotton (one pass) & 82.4 & 17.6 & 4.7 \\
rGO coated (padded) cotton (five passes) & 85.8 & 14.2 & 6.1 \\
\hline
\end{tabular}

rGO which display characteristic $D$ and $G$ peaks. After reduction, the intensity ratio of $\mathrm{D}$ to $\mathrm{G}$ band $\left(I_{\mathrm{D}} / I_{\mathrm{G}}\right)$ was increased from 0.98 for GO to 1.61 for rGO.

Scalable Production of Graphene E-Textiles. Padding is the most commonly used method in the textile industry to apply functional (e.g., water repellent, wrinkle free, moisture management, etc.) or soft finishes onto textiles. The production speed of a pad-dry unit is high and can potentially process approximately $150 \mathrm{~m}$ fabric in just $1 \mathrm{~min}$. In this study, a laboratory scale pad-dry unit was used to mimic an industrial pad-dry unit, which could potentially be used for large-scale industrial production of graphene e-textiles. Figure $3 a, d$ shows a white cotton control fabric which was de-sized, scoured, and bleached to remove natural and added impurities and colors. ${ }^{48}$ As shown in Figure 3a, this control fabric was passed through the padding bath which contains the rGO dispersion $(3.2 \mathrm{mg}$ / $\mathrm{mL}$ ), and the mangle nip rollers squeeze any excess rGO from the fabric surface, thus producing a uniform treatment. Almost immediately after coating (within few seconds), the white cotton fabrics turns black as shown in Figure $3 \mathrm{~b}$, due to the uniform deposition of rGO on the surface (Figure $3 \mathrm{~h}$ ) and also within each individual fiber. The rGO coated fabric was dried at $100{ }^{\circ} \mathrm{C}$ for $5 \mathrm{~min}$ in a Mathis laboratory dryer to dry off the water/solvent and fix the graphene onto the textiles.

Figure 3d,e shows untreated and rGO padded (five passes) cotton fabrics, respectively. Figure $3 \mathrm{f}$ demonstrates the excellent bendability and flexibility of the rGO padded cotton fabrics, which is similar to untreated cotton fabrics. The rGO coating on cotton fabric has not altered the "drape" (the way a fabric hangs under its own weight) of the fabrics. SEM images of the untreated cotton fabric, Figure $3 g$, show a typically smooth fiber, which clearly changed the morphology after deposition of the rGO flakes on the cotton fiber surface (Figure $3 \mathrm{~h}$ ). Unlike in the previously reported study where rGO was deposited between the fibers, ${ }^{19}$ in this study the rGO forms a uniform coating around the fibers. This is may be due to the smooth coating by padding and the hydrogen bonding between residual hydroxyl groups of rGO and cotton fibers. ${ }^{20,21}$ Therefore, rGO coated fabrics provide electric conductivity without compromising the breathability, flexibility, and comfort of the wearable e-textiles. Figure $3 \mathrm{~h}$ also shows some inter-fiber bonding between adjacent fibers, which may be due to the presence of the PSS cross-linker in the rGO dispersion. Figure $3 \mathrm{i}$ shows SEM images of washed (five times) rGO coated fabric, which appear almost unchanged even after the repeated washing cycles.

XPS analysis of the untreated and rGO coated cotton fabrics provides further evidence of efficient coating of rGO on the cotton fibers (Figure $4 \mathrm{a}, \mathrm{b}$ and Table 1 ). The wide scan XPS
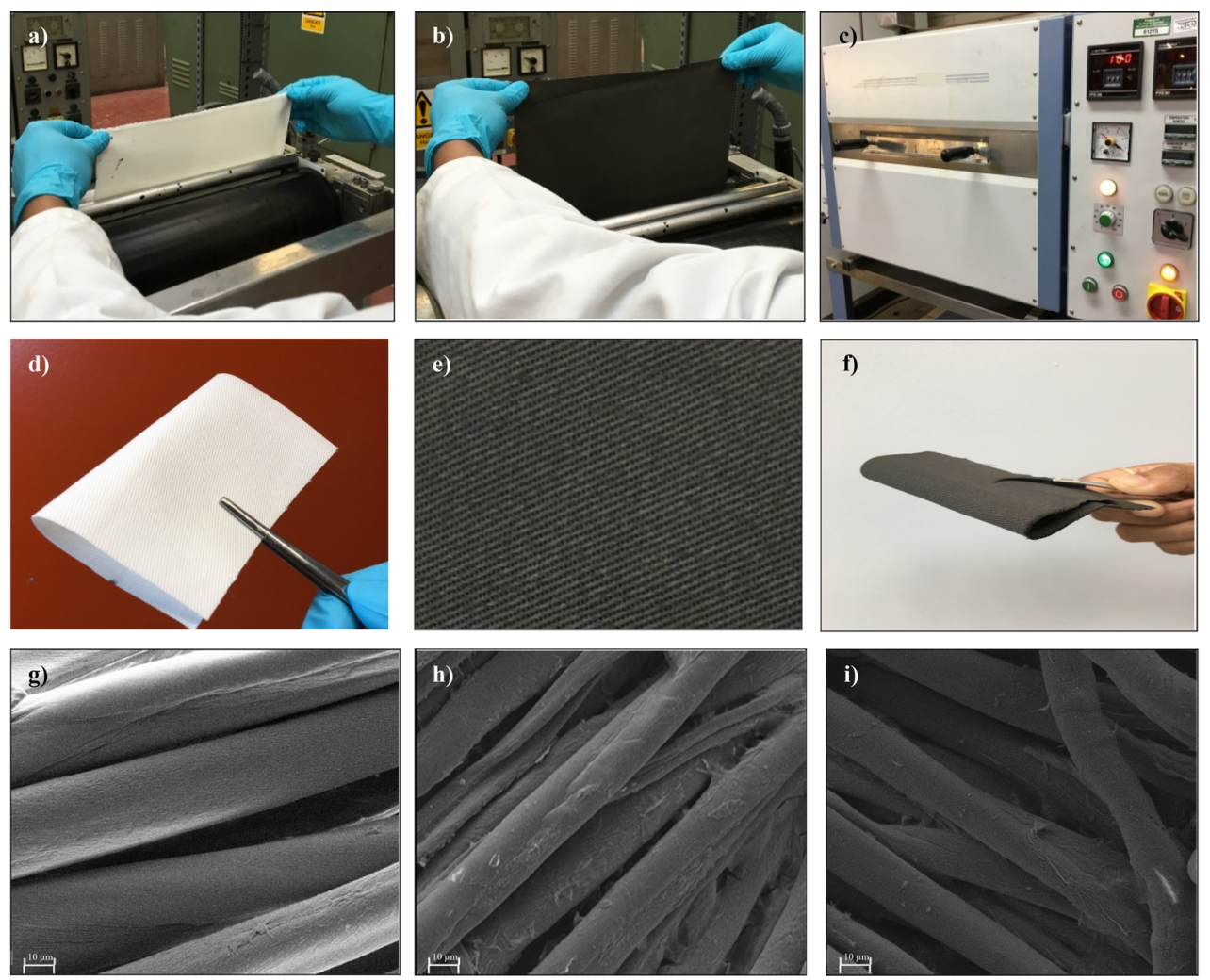

Figure 3. (a) Untreated cotton fabric was passed through padding bath and rollers. (b) rGO coated cotton fabric immediately after padding. (c) Drying of rGO coated cotton fabric at $100{ }^{\circ} \mathrm{C}$ for $5 \mathrm{~min}$. (d) Untreated control cotton fabric. (e) rGO coated cotton fabric after five padding passes and drying. (f) Demonstration of flexibility and drape of rGO coated cotton fabric. (g) SEM image of untreated control cotton fabrics $(\times 1000)$. (h) SEM image of rGO coated cotton fabric $(\times 1000)$. (i) SEM image of washed (five times) rGO coated cotton fabric $(\times 1000)$. 

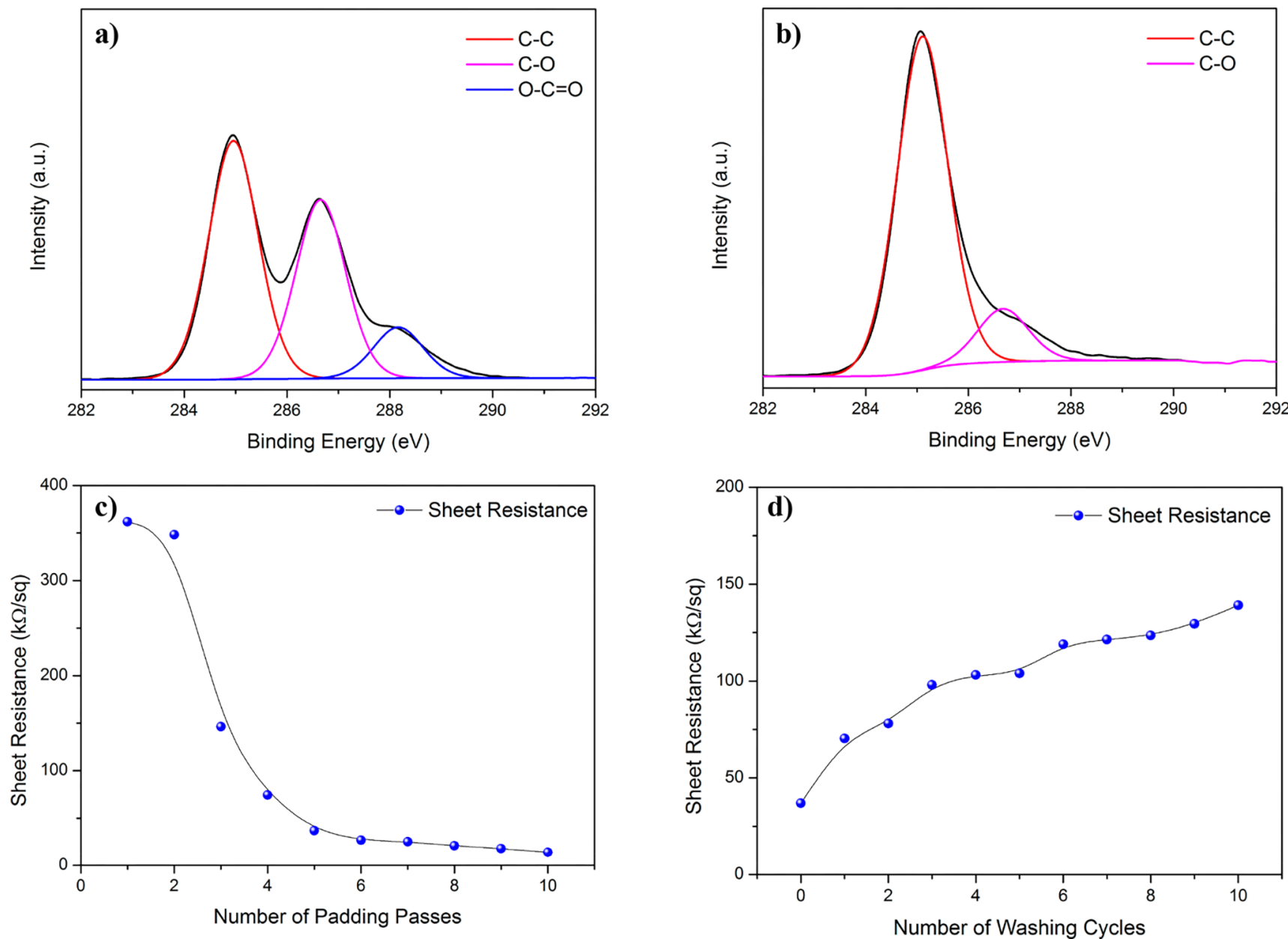

Figure 4. (a) High resolution C (1s) XPS spectrum of control cotton fabric. (b) High resolution C (1s) XPS spectrum of rGO padded cotton fabric. (c) Sheet resistance $v s$ number of padding passes. (d) The change of sheet resistance with number of washing cycles.

analysis shows an increase in carbon content from $73.9 \%$ for untreated cotton to $82.4 \%$ for rGO coated cotton (one pass) and a decrease in oxygen content from $26.1 \%$ for untreated cotton to $17.6 \%$ rGO coated cotton (one padding pass) (Table 1). Moreover, the $\mathrm{C} / \mathrm{O}$ ratio increased from 2.8 for untreated cotton to 4.7 for treated cotton (one padding pass). In addition, with the increase of number of padding passes, the $\mathrm{C} / \mathrm{O}$ ratio increased to 6.1 (for five padding passes). This was clearly due to the presence of more rGO flakes on the fiber surface at the higher number of padding passes. The $C$ (1s) spectrum of untreated cotton fabric in Figure $4 \mathrm{a}$ shows three main peaks that can be fitted into three components arising from $\mathrm{C}-\mathrm{C}$ bond $(\sim 284.6 \mathrm{eV}), \mathrm{C}-\mathrm{O}$ groups (hydroxyl, $\sim 286.2 \mathrm{eV}$ ), and $\mathrm{C}=\mathrm{O} / \mathrm{O}-\mathrm{C}-\mathrm{O}$ groups (carbonyl and acetal, $\sim 288 \mathrm{eV}){ }^{49}$ After coating with rGO, the peaks associated with the oxygen functional groups significantly diminished with small amounts of residual oxygen functional groups left around $287.5 \mathrm{eV}$ (Figure 4b).

Figure $4 \mathrm{c}$ shows the changes of sheet resistance of the $\mathrm{rGO}$ coated fabric with the number of padding passes. After one padding pass with $\mathrm{rGO}$, the sheet resistance of coated fabric was $361.82 \mathrm{k} \Omega / \mathrm{sq}$. There was a slight change in the sheet resistance of rGO coated cotton fabric up to two padding cycles. However, the sheet resistance then decreased sharply by $\sim 50-60 \%$ for each padding pass up to five padding passes. The sheet resistance of the rGO coated fabric decreased by $\sim 90 \%$ from $348.34 \mathrm{k} \Omega / \mathrm{sq}$ (after two padding passes) to $36.94 \mathrm{k} \Omega / \mathrm{sq}$ (after five padding passes). This could possibly be explained by an absorption and adsorption phenomena, where absorption of rGO dispersion into the fibers is pre-dominant in first few padding passes. Once it reached the saturation point, it is then mainly adsorbed on the fiber surface and formed a continuous conductive film by creating better connections between flakes. Thus, the sheet resistance of the fabric decreased by presenting more flakes on fiber surface (Figure $3 \mathrm{~h}$ ) and by the restacking of the flakes through the van der Waal forces applied by the squeeze rollers.

Figure $4 \mathrm{~d}$ shows the washability of the rGO coated fabric (five padding passes) after a number of washing cycles (1-10). The simulated standard washing conditions generated mechanical agitation and abrasion of the rGO coated fabric in the washing bath, which altered the continuity of conductive film on the fabric surfaces, resulting in an increase in sheet resistance. $^{19}$ The sheet resistance of $\mathrm{rGO}$ coated fabric increased significantly from $36.94 \mathrm{k} \Omega / \mathrm{sq}$ to $70.32 \mathrm{k} \Omega / \mathrm{sq}$ after the first washing cycle, probably be due to the removal of unfixed rGO flakes from the fabric surface. After that, the sheet resistance increased moderately to $139.09 \mathrm{k} \Omega / \mathrm{sq}$ after 10 washing cycles. We also examined various humidity conditions to observe its effect on the rGO coated cotton e-textiles; however, the complex impedance spectra (CIS) and $I-V$ curves (Figure S4a,b) showed that humidity had no effect on the impedance and resistance of rGO coated textiles, which may be due to the efficient reduction and hydrophobicity of the rGO. ${ }^{50}$ 

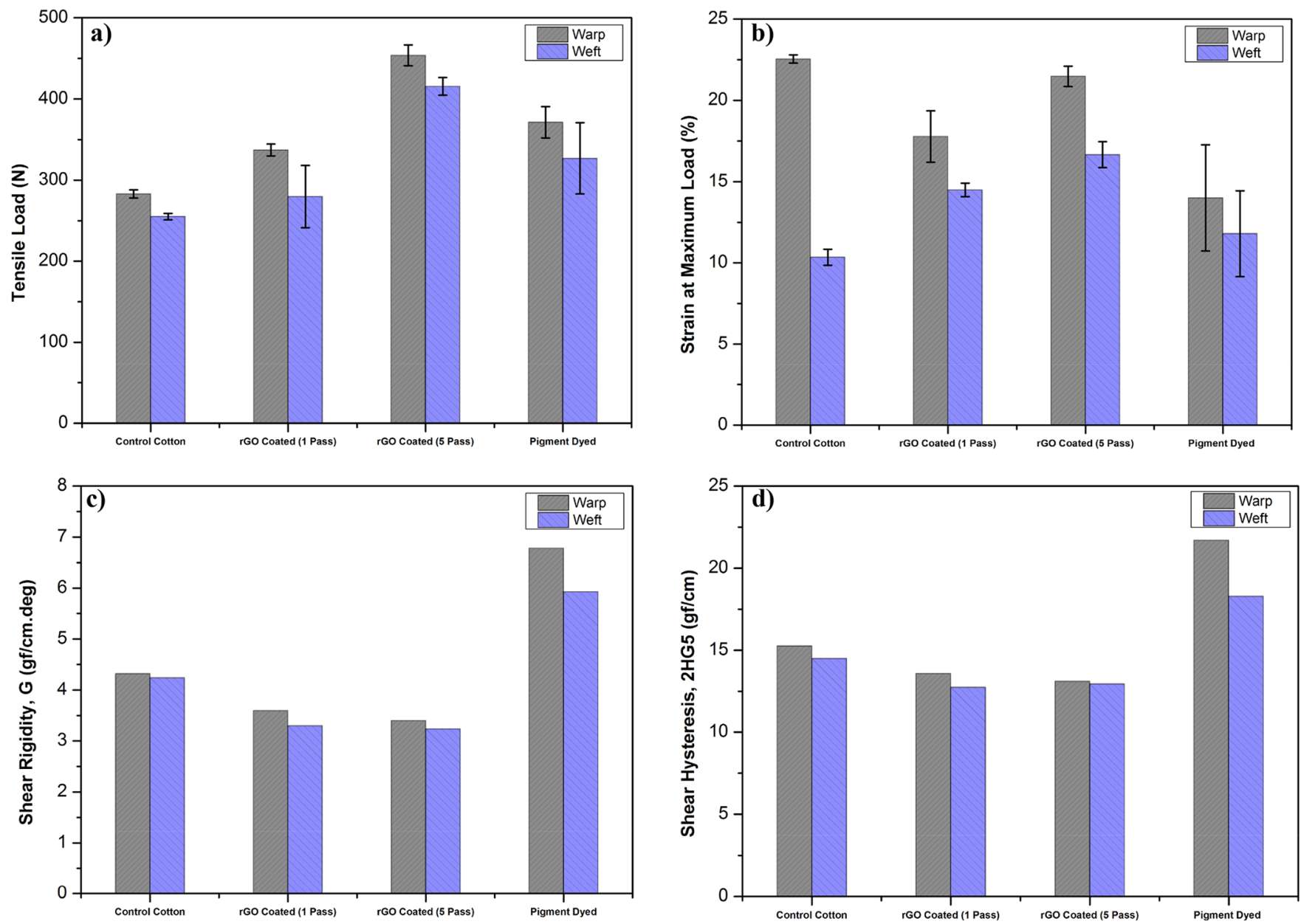

Figure 5. Effect of rGO coatings and pigment dyeing on (a) Tensile strength at maximum load and (b) Strain\% at maximum load. (c) Shear rigidity, $G$, and (d) Shear hysteresis at $5^{\circ}, 2 H G 5$, of cotton fabrics.

Almost similar graphs were observed for rGO coated cotton fabrics (with five padding passes) at $11 \%, 53 \%$, and $97 \%$ relative humidity.

The tensile strength of textile fabrics is one of the most important parameters that determines the quality and lifetime of a garment. Some of the chemical processing of cotton or cellulosic fibers seem to have significant negative effects on their tensile strength due to the damage in cellulosic structure. $^{51}$ Moreover, the higher curing temperature (above $140{ }^{\circ} \mathrm{C}$ ) for longer duration reduced the cotton fabric strength significantly due to the intra-macromolecular cross-linking and depolymerization of the cellulose. ${ }^{33}$ However, these issues were not addressed in previous studies on graphene-based e-textiles, where the toxic reducing agents such as hydrazine hydrate ${ }^{27}$ and a higher thermal reduction temperature for longer duration ${ }^{19}$ were used in the post-reduction process of GO coated textiles. Nevertheless, the lower or similar tensile strength achieved with chemically reduced GO coated textiles was reported to some extent. ${ }^{23}$ In contrast, we use an efficient and commonly used chemical $\left(\mathrm{Na}_{2} \mathrm{~S}_{2} \mathrm{O}_{4}\right)$ for textiles processing as the reducing agent and the relatively lower temperature (100 ${ }^{\circ} \mathrm{C}$ ) for shorter duration ( $5 \mathrm{~min}$ ) in our study. Figure 5a shows a significant increase in the tensile strength in both the warp and weft direction of the rGO coated cotton fabrics by $\sim 20 \%-$ $30 \%$ after a single padding pass, almost similar to pigment-dyed cotton fabrics and by $\sim 60 \%$ after five padding passes. Similarly, the elongation or strain\% at maximum load increased with rGO coatings showing enhancement in flexibility (displacement). ${ }^{52}$ These results are in agreement with previous studies, however, on rGO-based composites. ${ }^{53}$

The Kawabata evaluation system for fabrics (KES-F) is an objective measurement system based on tensile, shear, bending compression and surface analysis intruments that measure 16 mechanical parameters using non-destructive forces similar to those used by a human hand during typical fabric assessment and can characterize the fabric handle qualities (e.g., softness or stiffness, smoothness, etc.) perceived by human touch. ${ }^{54}$ In this study, the shear rigidity, $G$, and shear hysteresis, $2 H G 5$, of the rGO coated cotton fabrics (Figure $5 \mathrm{c}$,d) were measured, and it was found that the shear rigidity decreased after coating with rGO. While in contrast, the shear rigidity increased for the pigment-dyed fabrics due to the introduction of inter-fiber bonding by the cross-linking binder, which resulted in a stiffer and harsher hand feel. ${ }^{55}$ The shear hysteresis at $5^{\circ}, 2 \mathrm{HG} 5$, has previously been identified as the "best" indicator relating to subjective perception of fabric softness and is a sensitive measure of inter-yarn friction. ${ }^{56}$ If a processing treatment lubricates the fiber/yarn surface, such as a fabric softener, the 2 HG5 value decreases, while if the treatment roughens/ degrades, the surface $2 \mathrm{HGS}$ increases. In this case, the $2 \mathrm{HG5}$ value decreased with rGO coating, suggesting there was no formation of "restricting" inter-fiber bonds, but rather the rGO coating lubricates the fiber surfaces and results in an overall improvement in the fabric softness. In contrast, for pigment- 

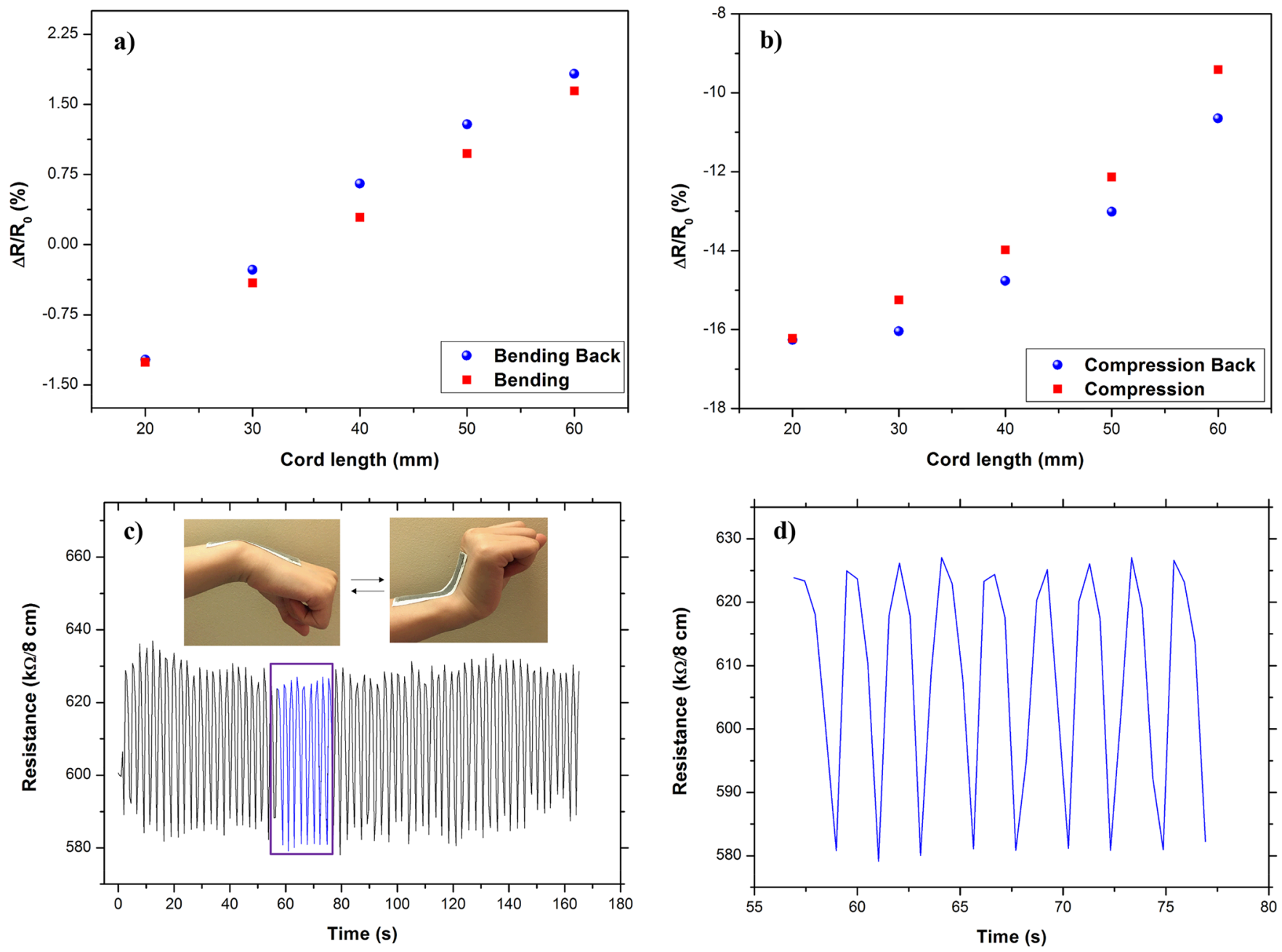

Figure 6. (a) The variation in resistance of the bending sensor in forward (bending) and reverse (bending back) direction. (b) The variation in resistance of the compression sensor in forward (compression) and reverse (compression back) direction. (c) The upward and downward movements of rGO coated cotton fabric mounted on a wrist joint. (d) Expanded version of purple box in (c) from $57 \mathrm{~s}$ to $77 \mathrm{~s}$.

dyed fabric the introduction of inter-fiber bonding in the fabric structure resulted in a stiffening/harshening of the fabric handle, and this was reflected in the much higher $2 H G 5$ values observed (Figure 5d).

Wearable Sensor Application. Figure 6a shows the changes in resistances per total length of the rGO coated fabric at various bending positions with concave down at various cord lengths (Figure S8) when the test is carried out on a tensile tester. Here, the cord length is defined as the distance between the tensile tester grips for the sensor material under investigation. As seen in Figure 6a, a repeatable response in forward (bending) and reverse (bending back) directions was observed. ${ }^{57}$ Similarly for compression with concave upward position, the change in resistance of the rGO coated cotton fabrics was repeatable in both the forward (compression) and reverse (compression back) directions (Figure 6b). Unlike in other studies, where the change in resistance of the coated or printed fabrics with bending (increase) and compression (decrease) was the opposite, ${ }^{19,58}$ here in both cases the resistance of the rGO coated fabric increased with the increase of cord length for bending and compression. This may be due to the fact that individual cotton fibers were uniformly coated with rGO during padding on both sides of the fabric and still maintained the same porosity of cotton woven fabric.
Moreover, strong connectivity between the flakes was achieved due to the pressure applied by padding rollers. Thus produced rGO coated fabric would provide better comfort and breathability to users. However, the change in resistance $\left(\Delta R / R_{0}\right)$ varied during bending and compression may be due to the different structure on the front and back side of the fabric.

In order to investigate the suitability of the rGO coated fabrics to be used as an activity monitoring sensor, for example, wrist movement monitoring, a piece of rGO coated (five padding passes) fabric was mounted on a wrist joint, Figure $6 c$, and the change in resistance per $8 \mathrm{~cm}$ length of the rGO coated fabric was continuously recorded during the wrist movement (i.e., when the sensor is mounted on the wrist, the sensor material on the wrist is bent and unbent cyclically). The sensory resistance data collected from the wrist mounted sensor are shown in Figure $6 \mathrm{c}, \mathrm{d}$. Figure $6 \mathrm{~d}$ shows the change in resistance due to the upward and downward movements of the wrist joint for a selected section of the total wrist activity recorded. The results show the capability of the rGO coated fabric to capture mechanical events such as bending/ unbending, stretching/relaxation, and twisting/untwisting. The woven cotton fabric showed the least variation in the electrical resistance over long period of cyclic tensile tests. 
Figure S11a,b shows that the woven cotton twill fabric has a different appearance on the front and back side of the fabric (Supporting Information). This imbalance in the surface structure caused the surface stress distributions on each side to vary. The strain\% of cotton fabric at the elastic limit is $2 \%$. Therefore, in order to ensure the least amount of plastic deformation of the fiber structure, the electro-mechanical performance of these fabrics was investigated within each respective elastic limit.

\section{CONCLUSIONS}

We report a simple and scalable production method for $\mathrm{rGO}$ dispersion and the coating of cotton textile fabrics with the rGO dispersion in order to produce electrically conductive graphene e-textiles. The rGO coated fabrics thus produced are washable, flexible, and bendable and exhibit a significant increase in the fabric tensile strength and strain\%. We also demonstrate the suitability of rGO coated fabric for applications as strain sensors to monitor human activities. We believe our scalable production method of producing graphenebased wearable e-textiles is an important step toward moving from R\&D-based e-textiles to actual real world applications.

\section{EXPERIMENTAL METHODS}

Materials. Flake graphite (Grade 3061) was supplied by Asbury Graphite Mills, USA. Poly(sodium 4-styrene sulfonate) (PSS, $M_{\mathrm{w}} \sim$ 70,000 , powder), sodium hydrosulphite $(\sim 82 \%)$, ammonia, potassium permanganate $\left(\mathrm{KMnO}_{4}\right)$, sulfuric acid $\left(\mathrm{H}_{2} \mathrm{SO}_{4}, \sim 99 \%\right)$, and hydrogen peroxide $\left(\mathrm{H}_{2} \mathrm{O}_{2}, \sim 30 \%\right)$ were purchased from Sigma-Aldrich, UK, and used as received. $100 \%$ Cotton 3/1 twill fabrics were manufactured internally in the University of Manchester weaving laboratory and desized, scoured, and bleached.

Synthesis of GO and rGO. Graphene oxide (GO) was prepared using a modified Hummers method as described elsewhere. ${ }^{37} \mathrm{~A}$ brown dispersion of GO $(1 \mathrm{mg} / \mathrm{mL})$ was prepared by adding $160 \mathrm{mg}$ of GO to $160 \mathrm{~mL}$ of deionized (DI) water and sonicated for $30 \mathrm{~min}$. In order to form a stable dispersion, $1.6 \mathrm{~g}$ of PSS was mixed into the GO dispersion by vigorous stirring. The resulting suspension was transferred to a round-bottom flask placed in an oil bath. $\mathrm{Na}_{2} \mathrm{~S}_{2} \mathrm{O}_{4}$ $(1.2 \mathrm{~g})$ and $\mathrm{NH}_{3}$ (required to adjust $\left.\mathrm{pH} 9-10\right)$ were added to the dispersion with vigorous stirring. This mixture was held at $90^{\circ} \mathrm{C}$ for 12 $\mathrm{h}$ under closed conditions in order to obtain a black dispersion. Any residual $\mathrm{Na}_{2} \mathrm{~S}_{2} \mathrm{O}_{4}$ and PSS were removed from the rGO by washing several times with deionized water and finally diluted into distilled water (DI) to adjust the rGO dispersion concentration to $3.2 \mathrm{mg} / \mathrm{mL}$.

Continuous Pad-Drying of Textiles with rGO Ink. Textile fabrics were padded one dip and one nip through rGO dispersions to a wet pick-up of $\sim 80 \%$ on the weight of the fabric (o.w.f.). The wet pick-up\% was calculated using following formula:

$$
\text { pick-up } \%=\frac{(\text { rGO coated fabric wt }- \text { untreated fabric dry wt })}{\text { untreated fabric dry wt }} \times 100 \%
$$

The rGO padded fabrics were subsequently dried at $100{ }^{\circ} \mathrm{C}$ for 10 min and were studied for e-textiles application. Also, several $\mathrm{rGO}$ coated samples were prepared using multiple $(1-10)$ padding passes to establish any improvement in the electrical conductivity of rGO coated fabrics. In order to asses with comparable textiles, a pigmentdyed cotton fabric sample was also prepared using a standard textile pigment dyeing recipe for $3 \%$ pigment black shade (recipe in Supporting Information and Table 1). The same padding method and parameters were used to apply pigments on the similar fabrics used for e-textiles.

Washability and Humidity Testing. The washability of the rGO coated cotton fabrics was assessed according to BS EN ISO 105 C06 A1S, by treating rGO coated fabrics in a solution containing $4 \mathrm{~g} / \mathrm{L}$ ECE reference detergent $\mathrm{B}$ and 10 stainless steel balls at $40{ }^{\circ} \mathrm{C}$ for 30 min. Ten steel balls were used to simulate the agitation and abrasion that a garment is subject to during a standard washing cycle. The fabrics were rinsed subsequently in running water at ambient temperature and air-dried at room temperature prior to further analysis.

The influence of humidity was measured by exposing the sample to a range of controlled relative humidity $(\mathrm{RH})$ levels, $11 \%, 53 \%$, and $97 \%$. To produce different $\mathrm{RH}$ environments, saturated aqueous solutions of $\mathrm{LiCl}$ and $\mathrm{K}_{2} \mathrm{SO} 4$ were prepared (Sigma-Aldrich) and placed in airtight glass vessels at a temperature of $24^{\circ} \mathrm{C}$, which yielded atmospheres with RHs of $11 \%$ and $97 \%$, respectively. The $53 \% \mathrm{RH}$ is the room RH level. The complex impedance spectra (CIS) and $I-V$ data of the sample were measured using a portable electrochemical interface and impedance analyzer (Ivium Technologies B.V., Eindhoven, Netherlands).

Materials Characterization. The sheet resistance of the conductive e-textiles was measured in van der Pouw geometry (a factor of 4.532 was used to obtain the sheet resistance from the raw measurements) using a Jandel four-probe system (Jandel Engineering Ltd., Leighton, UK). The mean sheet resistance was calculated from the average of six measurements. A Zeiss ultra scanning electron microscope (SEM) was used to analyze the surface topography of the untreated and rGO coated fabrics and also the flake size of GO and rGO. The dispersions were diluted 1000 times and drop-casted on $\mathrm{Si} /$ $\mathrm{SiO}_{2}$ (290 nm oxide on plain silicon) for each sample, and images were taken at 10 different locations on the sample. A dimension icon (Bruker) atomic force microscopy (AFM) was used to determine GO and rGO flake thickness. A Renishaw Raman system equipped with $633 \mathrm{~nm}$ laser was used to collect Raman spectra of GO and rGO flakes. A Kratos axis X-ray photoelectron spectroscopy (XPS) system was used to characterize the surface of untreated and rGO coated textiles and also the GO and rGO flakes.

Tensile strength and strain\% of untreated, rGO coated, and pigment-dyed cotton fabrics $(5 \times 2.5 \mathrm{~cm})$ were measured by a Zwick/ Roell tensile tester (Zwick Roell Group, Germany) at $200 \mathrm{~mm} / \mathrm{min}$ speed and calculated as an average from three measurements in both warp and weft directions of the fabrics. The Kawabata evaluation system for fabrics (KES-F) was used to determine the mechanical and surface properties of untreated and rGO coated ( 5 padding passes) fabric. ${ }^{54}$ The samples $(20 \times 20 \mathrm{~cm})$ were conditioned for $24 \mathrm{~h}$ at 20 ${ }^{\circ} \mathrm{C}$ and $65 \% \mathrm{RH}$ prior to analysis.

We used various cord lengths (Figures S8-S10) during bending (concave down) and compression (concave upward) to measure the change of resistance of rGO coated fabrics $(8 \times 1.5 \mathrm{~cm})$. The cord length of the rGO coated fabric was controlled on a Zwick/Roell tensile tester (Zwick Roell Group, Germany) during bending and compression tests both in the forward and reverse direction. The change of sheet resistance with the change of cord lengths of rGO coated fabric $(8 \times 1.5 \mathrm{~cm})$ during bending and compression was captured using a National Instrument 9219 data acquisition card (NI, American). A piece of rGO coated (5 pass) fabric $(8 \times 1.5 \mathrm{~cm})$ was also mounted on a wrist joint, and the changes in resistance of the rGO coated fabrics due to upward and downward movement were measured using a National Instrument 9219 card (NI, American).

\section{ASSOCIATED CONTENT}

\section{Supporting Information}

The Supporting Information is available free of charge on the ACS Publications website at DOI: 10.1021/acsnano.7b05921.

The formulation (recipe) for pigment dyeing; the chemical structures of the vat dyes and sodium hydrosulphite $\left(\mathrm{Na}_{2} \mathrm{~S}_{2} \mathrm{O}_{4}\right)$; images to show the efficient reduction of GO using $\mathrm{Na}_{2} \mathrm{~S}_{2} \mathrm{O}_{4}$; high-resolution XPS spectra of GO and rGO; the effect of humidity on the rGO coated textiles; SEM images of untreated, rGO coated, and washed rGO coated cotton fabrics; cord lengths; the change of resistance with bending and 
compression; and the front and reverse side appearance of coated cotton fabrics (PDF)

Demonstration of the capability of rGO coated fabric to capture mechanical events such as the resistance change during upward and downward movements of the wrist (MPG)

\section{AUTHOR INFORMATION}

\section{Corresponding Author}

*E-mail: mdnazmul.karim@manchester.ac.uk.

\section{ORCID}

Nazmul Karim: 0000-0002-4426-8995

\section{Author Contributions}

${ }^{\perp}$ These authors contributed equally.

\section{Notes}

The authors declare no competing financial interest.

\section{ACKNOWLEDGMENTS}

Authors kindly acknowledge the Government of Bangladesh for Ph.D. funding of S.A. and the National Graphene Institute, the University of Manchester. This work was supported by the EU Graphene Flagship Program, European Research Council Synergy Grant Hetero2D, The Royal Society, Engineering and Physical Sciences Research Council (EPSRC UK Grant Number: EP/N010345/1, 2015) and US Army Research Office (W911NF-16-1-0279). Authors also kindly acknowledge Dr. Ben Spencer, Dr. Chloe Holroyd, Daniel Wand, and Jianguo $\mathrm{Qu}$ for the help with XPS, AFM, graphics, and KES-F, respectively.

\section{REFERENCES}

(1) De Rossi, D. Electronic Textiles: A Logical Step. Nat. Mater. 2007, 6, 328-329.

(2) Service, R. F. Electronic Textiles Charge Ahead. Science 2003, 301, 909-911.

(3) E-Textiles 2017-2027: Technologies, Markets, Players; IDTechEX: Cambridge, UK, 2017

(4) Xu, L.; Yang, G.; Jing, H.; Wei, J.; Han, Y. Ag-Graphene Hybrid Conductive Ink for Writing Electronics. Nanotechnology 2014, 25, 055201.

(5) Sondergaard, R. R; Espinosa, N.; Jorgensen, M.; Krebs, F. C. Efficient Decommissioning and Recycling of Polymer Solar Cells: Justification for Use of Silver. Energy Environ. Sci. 2014, 7, 1006-1012.

(6) Irimia-Vladu, M.; Głowacki, E. D.; Voss, G.; Bauer, S.; Sariciftci, N. S. Green and Biodegradable Electronics. Mater. Today 2012, 15, 340-346.

(7) Novoselov, K. S.; Geim, A. K.; Morozov, S. V.; Jiang, D.; Zhang, Y.; Dubonos, S. V.; Grigorieva, I. V.; Firsov, A. A. Electric Field Effect In Atomically Thin Carbon Films. Science 2004, 306, 666-669.

(8) Geim, A. K. Graphene: Status and Prospects. Science 2009, 324, 1530-1534.

(9) He, Q.; Wu, S.; Yin, Z.; Zhang, H. Graphene-Based Electronic Sensors. Chem. Sci. 2012, 3, 1764-1772.

(10) Li, D.; Müller, M. B.; Gilje, S.; Kaner, R. B.; Wallace, G. G. Processable Aqueous Dispersions of Graphene Nanosheets. Nat. Nanotechnol. 2008, 3, 101-105.

(11) Stankovich, S.; Dikin, D. A.; Dommett, G. H. B.; Kohlhaas, K. M.; Zimney, E. J.; Stach, E. A.; Piner, R. D.; Nguyen, S. T.; Ruoff, R. S. Graphene-Based Composite Materials. Nature 2006, 442, 282-286.

(12) Stankovich, S.; Dikin, D. A.; Piner, R. D.; Kohlhaas, K. A.; Kleinhammes, A.; Jia, Y.; Wu, Y.; Nguyen, S. T.; Ruoff, R. S. Synthesis of Graphene-Based Nanosheets via Chemical Reduction of Exfoliated Graphite Oxide. Carbon 2007, 45, 1558-1565.
(13) Kim, J.; Cote, L. J.; Kim, F.; Yuan, W.; Shull, K. R.; Huang, J. Graphene Oxide Sheets at Interfaces. J. Am. Chem. Soc. 2010, 132, $8180-8186$

(14) Li, D.; Muller, M. B.; Gilje, S.; Kaner, R. B.; Wallace, G. G. Processable Aqueous Dispersions of Graphene Nanosheets. Nat. Nanotechnol. 2008, 3, 101-105.

(15) Paredes, J. I.; Villar-Rodil, S.; Martínez-Alonso, A.; Tascón, J. M. D. Graphene Oxide Dispersions in Organic Solvents. Langmuir 2008, 24, 10560-10564.

(16) Yu, G.; Hu, L.; Vosgueritchian, M.; Wang, H.; Xie, X.; McDonough, J. R.; Cui, X.; Cui, Y.; Bao, Z. Solution-Processed Graphene $/ \mathrm{MnO}_{2}$ Nanostructured Textiles for High-Performance Electrochemical Capacitors. Nano Lett. 2011, 11, 2905-2911.

(17) Liu, L.; Yu, Y.; Yan, C.; Li, K.; Zheng, Z. Wearable EnergyDense and Power-Dense Supercapacitor Yarns Enabled by Scalable Graphene-Metallic Textile Composite Electrodes. Nat. Commun. 2015, 6, 7260.

(18) Shateri-Khalilabad, M.; Yazdanshenas, M. E. Preparation of Superhydrophobic Electroconductive Graphene-Coated Cotton Cellulose. Cellulose 2013, 20, 963-972.

(19) Ren, J.; Wang, C.; Zhang, X.; Carey, T.; Chen, K.; Yin, Y.; Torrisi, F. Environmentally-Friendly Conductive Cotton Fabric as Flexible Strain Sensor Based on Hot Press Reduced Graphene Oxide. Carbon 2017, 111, 622-630.

(20) Abdelkader, A. M.; Karim, N.; Vallés, C.; Afroj, S.; Novoselov, K. S.; Yeates, S. G. Ultraflexible and Robust Graphene Supercapacitors Printed on Textiles for Wearable Electronics Applications. 2D Mater. 2017, 4, 035016.

(21) Karim, N.; Afroj, S.; Malandraki, A.; Butterworth, S.; Beach, C.; Rigout, M.; Novoselov, K.; Casson, A. J.; Yeates, S. All Inkjet-Printed Graphene-Based Conductive Patterns for Wearable E-Textile Applications. J. Mater. Chem. C 2017, 5, 11640.

(22) Molina, J.; Fernández, J.; Inés, J. C.; del Río, A. I.; Bonastre, J.; Cases, F. Electrochemical Characterization of Reduced Graphene Oxide-Coated Polyester Fabrics. Electrochim. Electrochim. Acta 2013, $93,44-52$.

(23) Shateri-Khalilabad, M.; Yazdanshenas, M. E. Fabricating Electroconductive Cotton Textiles Using Graphene. Carbohydr. Polym. 2013, 96, 190-195.

(24) Tang, X.; Tian, M.; Qu, L.; Zhu, S.; Guo, X.; Han, G.; Sun, K.; $\mathrm{Hu}, \mathrm{X}$.; Wang, Y.; Xu, X. Functionalization of Cotton Fabric With Graphene Oxide Nanosheet and Polyaniline for Conductive and UV Blocking Properties. Synth. Met. 2015, 202, 82-88.

(25) Javed, K.; Galib, C. M. A.; Yang, F.; Chen, C. M.; Wang, C. A New Approach to Fabricate Graphene Electro-Conductive Networks on Natural Fibers by Ultraviolet Curing Method. Synth. Met. 2014, 193, 41-47.

(26) Cao, Y.; Zhu, M.; Li, P.; Zhang, R.; Li, X.; Gong, Q.; Wang, K.; Zhong, M.; Wu, D.; Lin, F.; Zhu, H. Boosting Supercapacitor Performance of Carbon Fibres Using Electrochemically Reduced Graphene Oxide Additives. Phys. Chem. Chem. Phys. 2013, 15, 1955019556.

(27) Maiti, U. N.; Maiti, S.; Das, N. S.; Chattopadhyay, K. K. Hierarchical Graphene Nanocones Over 3D Platform of Carbon Fabrics: A Route Towards Fully Foldable Graphene Based Electron Source. Nanoscale 2011, 3, 4135-4141.

(28) Woltornist, S. J.; Alamer, F. A.; McDannald, A.; Jain, M.; Sotzing, G. A.; Adamson, D. H. Preparation of Conductive Graphene/ Graphite Infused Fabrics Using an Interface Trapping Method. Carbon 2015, 81, 38-42.

(29) Neves, A. I. S.; Bointon, T. H.; Melo, L. V.; Russo, S.; de Schrijver, I.; Craciun, M. F.; Alves, H. Transparent Conductive Graphene Textile Fibers. Sci. Rep. 2015, 5, 9866.

(30) Skrzetuska, E.; Puchalski, M.; Krucińska, I. Chemically Driven Printed Textile Sensors Based on Graphene and Carbon Nanotubes. Sensors 2014, 14, 16816.

(31) Samad, Y. A.; Li, Y.; Alhassan, S. M.; Liao, K. Non-Destroyable Graphene Cladding an a Range of Textile and Other Fibers and Fiber Mats. RSC Adv. 2014, 4, 16935-16938. 
(32) Ramadoss, A.; Saravanakumar, B.; Kim, S. J. Thermally Reduced Graphene Oxide-Coated Fabrics for Flexible Supercapacitors and SelfPowered Systems. Nano Energy 2015, 15, 587-597.

(33) Xu, W.; Li, Y. Cotton Fabric Strength Loss From Treatment with Polycarboxylic Acids for Durable Press Performance. Text. Res. J. 2000, 70, 957-961.

(34) Ju Yun, Y.; Hong, W. G.; Choi, N. J.; Hoon Kim, B.; Jun, Y.; Lee, H. K. Ultrasensitive and Highly Selective Graphene-Based Single Yarn for Use in Wearable Gas Sensor. Sci. Rep. 2015, 5, 10904.

(35) Gao, J.; Liu, F.; Liu, Y.; Ma, N.; Wang, Z.; Zhang, X. Environment-Friendly Method to Produce Graphene that Employs Vitamin C and Amino Acid. Chem. Mater. 2010, 22, 2213-2218.

(36) Hummers, W. S.; Offeman, R. E. Preparation of Graphitic Oxide. J. Am. Chem. Soc. 1958, 80, 1339-1339.

(37) Rourke, J. P.; Pandey, P. A.; Moore, J. J.; Bates, M.; Kinloch, I. A.; Young, R. J.; Wilson, N. R. The Real Graphene Oxide Revealed: Stripping the Oxidative Debris from the Graphene-Like Sheets. Angew. Chem. 2011, 123, 3231-3235.

(38) Zhong, Y. L.; Tian, Z.; Simon, G. P.; Li, D. Scalable Production of Graphene via Wet Chemistry: Progress And Challenges. Mater. Today 2015, 18, 73-78.

(39) Yoe, J. H. The Reduction of Certain Vat Dyes By Means of Alkaline Sodium Hydrosulphite. J. Phys. Chem. 1923, 28, 1211-1217.

(40) Zhou, T.; Chen, F.; Liu, K.; Deng, H.; Zhang, Q.; Feng, J.; Fu, Q. A Simple and Efficient Method to Prepare Graphene by Reduction of Graphite Oxide with Sodium Hydrosulfite. Nanotechnology 2011, 22, 045704 .

(41) Zhou, T.; Chen, F.; Tang, C.; Bai, H.; Zhang, Q.; Deng, H.; Fu, Q. The Preparation of High Performance and Conductive Poly (Vinyl Alcohol)/Graphene Nanocomposite via Reducing Graphite Oxide with Sodium Hydrosulfite. Compos. Sci. Technol. 2011, 71, 1266-1270.

(42) Liu, J.; Tang, J.; Gooding, J. J. Strategies for Chemical Modification of Graphene and Applications of Chemically Modified Graphene. J. Mater. Chem. 2012, 22, 12435-12452.

(43) Lonkar, S. P.; Deshmukh, Y. S.; Abdala, A. A. Recent Advances in Chemical Modifications of Graphene. Nano Res. 2015, 8, 10391074.

(44) Stankovich, S.; Piner, R. D.; Chen, X.; Wu, N.; Nguyen, S. T.; Ruoff, R. S. Stable Aqueous Dispersions of Graphitic Nanoplatelets via the Reduction of Exfoliated Graphite Oxide in the Presence of Poly (Sodium 4-Styrenesulfonate). J. Mater. Chem. 2006, 16, 155-158.

(45) Wei, D.; Li, H.; Han, D.; Zhang, Q.; Niu, L.; Yang, H.; Bower, C.; Andrew, P.; Ryhänen, T. Properties of Graphene Inks Stabilized by Different Functional Groups. Nanotechnology 2011, 22, 245702.

(46) Abdelkader, A.; Kinloch, I.; Dryfe, R. High-Yield ElectroOxidative Preparation of Graphene Oxide. Chem. Commun. 2014, 50, 8402-8404.

(47) Wang, T.; Li, Y.; Geng, S.; Zhou, C.; Jia, X.; Yang, F.; Zhang, L.; Ren, X.; Yang, H. Preparation of Flexible Reduced Graphene Oxide/ Poly(Vinyl Alcohol) Film with Superior Microwave Absorption Properties. RSC Adv. 2015, 5, 88958-88964.

(48) Tomasino, C. Chemistry \& Technology of Fabric Preparation \& Finishing. North Carolina State University: NC, 1992.

(49) Shang, S. M.; Li, Z.; Xing, Y.; Xin, J. H.; Tao, X. M. Preparation of Durable Hydrophobic Cellulose Fabric from Water Glass And Mixed Organosilanes. Appl. Surf. Sci. 2010, 257, 1495-1499.

(50) Su, Y.; Kravets, V. G.; Wong, S. L.; Waters, J.; Geim, A. K.; Nair, R. R. Impermeable Barrier Films and Protective Coatings Based on Reduced Graphene Oxide. Nat. Commun. 2014, 5, 4843.

(51) Powers, D. Effect of Synthetic Resins on Cellulose and Protein Fibers. Ind. Eng. Chem. 1945, 37, 188-193.

(52) Liu, Y.; Wang, X.; Qi, K.; Xin, J. Functionalization of Cotton with Carbon Nanotubes. J. Mater. Chem. 2008, 18, 3454-3460.

(53) Li, Z.; Guo, Q.; Li, Z.; Fan, G.; Xiong, D. B.; Su, Y.; Zhang, J.; Zhang, D. Enhanced Mechanical Properties of Graphene (Reduced Graphene Oxide)/Aluminum Composites with a Bioinspired Nanolaminated Structure. Nano Lett. 2015, 15, 8077-8083.

(54) Kawabata, S.; Niwa, M. Fabric Performance in Clothing and Clothing Manufacture. J. Text. Inst. 1989, 80, 19-50.
(55) Karim, M. N.; Rigout, M.; Yeates, S. G.; Carr, C. Surface Chemical Analysis of the Effect of Curing Conditions on the Properties of Thermally-Cured Pigment Printed Poly (Lactic Acid) Fabrics. Dyes Pigm. 2014, 103, 168-174.

(56) Finnimore, E.; Konig, A. Instrumental Assessment of Softening Effects on Fabrics: I. Correlation between Instrumental Measurements and Manual Assessments. Melliand Textilber. 1986, 67, 514-516.

(57) Cheng, Y.; Wang, R.; Sun, J.; Gao, L. A Stretchable and Highly Sensitive Graphene-Based Fiber for Sensing Tensile Strain, Bending, and Torsion. Adv. Mater. 2015, 27, 7365-7371.

(58) Liao, X.; Liao, Q.; Yan, X.; Liang, Q.; Si, H.; Li, M.; Wu, H.; Cao, S.; Zhang, Y. Flexible and Highly Sensitive Strain Sensors Fabricated by Pencil Drawn for Wearable Monitor. Adv. Funct. Mater. 2015, 25, 2395-2401. 